\title{
Nuclear magnetic resonance-based serum metabolic profiling of dairy cows with footrot
}

\author{
Jiasan $\mathrm{ZHENG}^{1,2) \# \text {, Lingwei SUN }}{ }^{3) \#, ~ S h i ~ S H U 2), ~ K u i l i n g ~} \mathrm{ZHU}^{2)}$, Chuang $\mathrm{XU}^{2)}$, Junsong $\mathrm{WANG}^{4)}$ and Hongbin $\mathrm{WANG}^{1) *}$ \\ ${ }^{1)}$ College of Veterinary Medicine, Northeast Agricultural University, 59 Mucai Street, Harbin 150030, P.R. China \\ ${ }^{2)}$ College of Animal Science and Veterinary Medicine, Heilongjiang Bayi Agricultural University, Daqing High-tech Industrial \\ Development Zone, Daqing 163319, P.R. China \\ 3) Jiangsu Engineering Technology Research Center of Meat Sheep \& Goat Industry, Nanjing Agricultural University, Nanjing, Jiangsu \\ 210095, P.R. China \\ 4)Center for Molecular Metabolism, School of Environmental and Biological Engineering, Nanjing University of Science Technology, \\ Nanjing 210095, P.R. China
}

(Received 10 January 2016/Accepted 10 May 2016/Published online in J-STAGE 18 June 2016)

ABSTRACT. Footrot is a debilitating and contagious disease in dairy cows, caused by the Gram-negative anaerobe Dichelobacter nodosus. ${ }^{1} \mathrm{H}-\mathrm{NMR}$ (nuclear magnetic resonance)-based metabolomics has been previously used to understand the pathology and etiology of several diseases. The objective of this study was to characterize serum from dairy cows with footrot $(\mathrm{n}=10)$ using ${ }^{1} \mathrm{H}-\mathrm{NMR}$-based metabolomics and chemometric analyses. ${ }^{1} \mathrm{H}-\mathrm{NMR}$ spectroscopy with multivariate pattern recognition (principal component analysis and orthogonal partial least-squares discriminant analysis) was performed to identify biomarkers in cows with footrot (F) and healthy controls (C). ${ }^{1} \mathrm{H}-$ NMR analysis facilitated the identification of 21 metabolites. Among these metabolites, 4 metabolites were higher and 17 metabolites were lower in the $\mathrm{F}$ group than in the $\mathrm{C}$ group. The serum levels of 5 metabolites were significantly different $(P<0.05)$ between the two groups. The results revealed that cows with footrot have altered carbohydrate, amino acid, lipid and energy metabolic pathways. Metabolomic approaches are a clinically useful diagnostic tool for understanding the biochemical alterations and mechanisms of several diseases.

KEY WORDS: ${ }^{1} \mathrm{H}-\mathrm{NMR}$, dairy cow, footrot, metabolomics, pathway

doi: 10.1292/jvms.15-0720; J. Vet. Med. Sci. 78(9): 1421-1428, 2016

Footrot is a debilitating and contagious disease in ruminants that is caused by Dichelobacter nodosus, a Gramnegative anaerobe $[6,23]$. Footrot, characterized by odorous exudative inflammation and keratinous hoof separation, is associated with anorexia and reduced milk yield in cows [17]. This painful condition causes lameness due to swelling and purulence of the foot, cracking and sloughing of interdigital skin, and puffiness of the coronary band. Infectious, immune, hormonal, nutritional and environmental factors are involved in footrot pathogenesis [16]. Footrot, which has an average worldwide incidence rate of $15-25 \%$ in dairy cows, causes significant financial losses in the dairy and beef cattle industries owing to expensive preventive and therapeutic interventions, reduced animal welfare and decreased productivity [37].

Current footrot diagnostic procedures include clinical examination, in vitro agglutination tests and polymerase chain reaction (PCR) [8]. If footrot control and management were effective during the non-transmission period, the dis-

\footnotetext{
*Correspondence to: Wang, H., College of Veterinary Medicine, Northeast Agricultural University, 59 Mucai Street, Harbin 150030, P.R. China. e-mail: hbwang@neau.edu.cn

\#These authors contributed equally to this work and should be considered co-first authors.

(C)2016 The Japanese Society of Veterinary Science

This is an open-access article distributed under the terms of the Creative Commons Attribution Non-Commercial No Derivatives (by-nc-nd) License $<$ http://creativecommons.org/licenses/by-nc-nd/4.0/>.
}

ease could be completely eradicated. Currently, the complex pathogenesis of the disease poses considerable challenges to diagnosis and monitoring.

Fields, such as systems biology, genomics, transcriptomics, proteomics and metabolomics, have facilitated the development of novel diagnostic technologies. Serum proteomic analyses of cows infected with footrot represent an effective method for disease diagnosis [27]. However, proteomic analyses fail to reveal the complete metabolic profile of an animal. On the other hand, metabolomics measures the metabolic responses to genetic modification or pathophysiological stimuli [24]. Additionally, metabolomics complements information obtained from genetic and proteomic analyses. Currently, metabolomic techniques include nuclear magnetic resonance (NMR) spectroscopy, mass spectrometry and metabolite chips. Among these techniques, ${ }^{1} \mathrm{H}-\mathrm{NMR}$ has been frequently used to assess the role of metabolites in the body and their association with various pathological conditions. Recently, ${ }^{1} \mathrm{H}-\mathrm{NMR}$ has been used to investigate the effects of fatty liver disease, ketosis, milk fever and displaced abomasum on cow metabolism [3, 28, 29, 35]. However, the biochemical changes that occur in cows with footrot are unknown.

The main objective of this study was to use ${ }^{1} \mathrm{H}-\mathrm{NMR}-$ based metabolomics to assess metabolic differences between dairy cows with footrot and healthy controls. This study will provide useful information on footrot metabolite biomarkers. 


\section{MATERIALS AND METHODS}

Ethics statement: This study was conducted in accordance with the Guide for the Care and Use of Laboratory Animals. The study was approved by the Ethics Committee of Heilongjiang Bayi Agricultural University (Permit Number: 20120319-1).

Animals: A commercial Holstein dairy cow farm in China suffering from an outbreak of footrot was chosen for this study. The cows in this study were at the same age and parity (1-3 births and 3-5 years old), in the middle stage of lactation. Cows acutely affected by footrot $(n=10)$ were assigned to a test group ( $\mathrm{F}$ group), while healthy cows $(n=10)$ were assigned to the control group (C group). Footrot diagnosis was based on the presence of lameness in one or more feet and swollen or necrotic skin in the digits. Cows with severe lameness, commonly attributed to other diseases (e.g., foot and toe abscesses, white line disease and foot-and-mouth disease) were excluded from the study. Similarly, cows with mastitis, respiratory system diseases and digestive system diseases were excluded. Definitive footrot diagnosis was achieved by PCR (results not shown). To examine healthy cows, control samples from unaffected cattle in the affected dairy herd were pooled using the same procedure. All cows in this study were fed a total mixed ration (TMR) at 05:00, 14:00 and 20:00 hr. The TMR consisted of 55.60\% dry matter (DM), $16.00 \%$ crude protein, $34.30 \%$ neutral detergent fiber, $22.00 \%$ acid detergent fiber, $5.60 \%$ fat, $1.07 \%$ calcium, $0.49 \%$ phosphorus, $0.32 \%$ magnesium, $0.13 \%$ sodium, $1.40 \%$ potassium, $0.39 \%$ chloride and $0.22 \%$ sulfur [28]. Table 1 shows the age, parity, dry matter intake (DMI) and body condition score (BCS) of cows in this study. The day before blood sample collection, the food intake of cows used for this study was recorded. BCS consists of a five-point scale (1 represents thin, and 5 represents obese) commonly used to assess body fat stores in Holstein dairy cows [34].

Sample collection and preparation: Following an overnight fast, blood samples $(10 \mathrm{~m} l)$ were collected from the vena caudalis between 06:00 and 07:00 $\mathrm{hr}$ and centrifuged $\left(3,500 \times \mathrm{g}, 20 \mathrm{~min}\right.$ at $\left.4^{\circ} \mathrm{C}\right)$ to obtain the serum. The resulting serum samples were transferred into $1.5 \mathrm{~m} l$ Eppendorf tubes and stored at $-80^{\circ} \mathrm{C}$.

Biochemical analyses: Serum levels of glucose (Glc), triglycerides (TG), total protein (TP), aspartate aminotransferase (AST) and alanine aminotransferase (ALT) were measured with a Beckman Synchron CX5CE analyzer (Beckman Coulter, Inc., Fullerton, CA, U.S.A.). Commercial kits (Diagnostic Product, Nanjing Jiancheng Bioengineering Institute, Nanjing, China) were used to analyze serum nitric oxide (NO) level and activities of glutathione peroxidase (GSH-Px) and superoxide dismutase (SOD). All kits were utilized according to the manufacturer's recommendations. Serum maleic dialdehyde (MDA) concentration was determined by the double heating method [10]. Serum total antioxidant capacity (TAC) was determined by performing a thiobarbituric acid reactive substances radical cation colorization assay as described by Guzel et al. [13].

Serum metabolic profiling by ${ }^{1} H$-NMR: Serum was al-
Table 1. Clinical data and biochemical parameters of healthy cows (C) and cows with footrot $(\mathrm{F})$

\begin{tabular}{clrcc}
\hline No. & Parameters & \multicolumn{1}{c}{ C group } & \multicolumn{1}{c}{ F group } & $P$-values \\
\hline 1 & Number & \multicolumn{1}{c}{10} & \multicolumn{1}{c}{10} & - \\
2 & Age $(\mathrm{yr})$ & $3.98 \pm 0.26$ & $3.82 \pm 0.24$ & 0.651 \\
3 & Parity & $1.90 \pm 0.23$ & $2.10 \pm 0.23$ & 0.550 \\
4 & BCS & $3.30 \pm 0.10$ & $2.72 \pm 0.11$ & 0.001 \\
5 & DMI $(\mathrm{kg} /$ day $)$ & $18.07 \pm 0.28$ & $17.23 \pm 0.31$ & 0.058 \\
6 & Glc $\left(\mathrm{mmol} \times l^{-1}\right)$ & $3.01 \pm 0.28$ & $2.68 \pm 0.08$ & 0.026 \\
7 & TG $\left(\mathrm{mmol} \times l^{-1}\right)$ & $0.70 \pm 0.04$ & $0.68 \pm 0.03$ & 0.665 \\
8 & ALT $\left(\mathrm{U} \times l^{-1}\right)$ & $20.07 \pm 0.53$ & $20.92 \pm 0.58$ & 0.297 \\
9 & AST $\left(\mathrm{U} \times l^{-1}\right)$ & $70.76 \pm 1.62$ & $71.49 \pm 1.80$ & 0.770 \\
10 & TP $\left(\mathrm{g} \times l^{-1}\right)$ & $62.54 \pm 1.55$ & $61.49 \pm 1.20$ & 0.581 \\
11 & NO $\left(\mu \mathrm{mol} \times l^{1}\right)$ & $1.86 \pm 0.18$ & $4.59 \pm 0.78$ & $<0.001$ \\
12 & MDA $\left(\mathrm{nmol} \times l^{-1}\right)$ & $5.39 \pm 0.22$ & $6.23 \pm 0.37$ & 0.077 \\
13 & T-AOC $\left(\mathrm{U} \times \mathrm{m} l^{-1}\right)$ & $48.17 \pm 1.19$ & $40.74 \pm 0.65$ & $<0.001$ \\
14 & SOD $\left(\mathrm{U} \times \mathrm{m} l^{-1}\right)$ & $108.66 \pm 2.90$ & $124.38 \pm 2.31$ & $<0.001$ \\
15 & GSH-Px $\left(\mathrm{U} \times l^{-1}\right)$ & $292.23 \pm 2.29$ & $245.81 \pm 2.62$ & $<0.001$ \\
\hline
\end{tabular}

a) Data are expressed as mean \pm SEM where appropriate. Abbreviations: BCS (body condition score), Glc (glucose), TG (triglycerides), ALT (alanine aminotransferase), AST (aspartate aminotransferase), TP (total protein), NO (nitric oxide), MDA (maleic dialdehyde), T-AOC (total antioxidant capacity), SOD (activities of superoxide dismutase) and GSH-Px (glutathione peroxidase).

lowed to thaw at room temperature and was centrifuged at $10,000 \times \mathrm{g}$ for $10 \mathrm{~min}$ at $4{ }^{\circ} \mathrm{C}$. An aliquot $(200 \mu l)$ of the resulting supernatant was mixed with $150 \mu l$ buffer $(\mathrm{pH} 7.4$, $0.2 \mathrm{~mol} / l \mathrm{Na}_{2} \mathrm{HPO}_{4}$ and $0.2 \mathrm{~mol} / l \mathrm{NaH}_{2} \mathrm{PO}_{4}$ ) and $150 \mu l$ sodium 3-trimethylsilyl-(2,2,3,3-D4) propionate (TSP, $1 \mathrm{mg} /$ $\mathrm{ml}$, Sigma-Aldrich, St. Louis, MO, U.S.A.). Subsequently, the samples were centrifuged again at $10,000 \times \mathrm{g}$ for $10 \mathrm{~min}$ at $4^{\circ} \mathrm{C}$. Finally, $550 \mu \mathrm{l}$ of the resulting supernatant was transferred into $5 \mathrm{~mm}$ NMR tubes containing $100 \mu l \mathrm{D}_{2} \mathrm{O}$.

NMR was performed at $25^{\circ} \mathrm{C}$ in a Bruker Avance spectrometer with a ${ }^{1} \mathrm{H}$ operating frequency of $500 \mathrm{MHz}$ (Bruker, Ettlingen, Germany). One-dimensional (1D) spectra were recorded with the Carr-Purcell-Meiboom-Gill (CPMG) sequence of $\mathrm{D}-\left[-90^{\circ}-\left(\tau-180^{\circ}-\tau\right) \mathrm{n}\right.$-acquisition], with a total spin-echo relaxation delay $(2 \mathrm{n} \tau)$ of $10 \mathrm{msec}$. Free induction decays (FIDs) were obtained with 128 scans of $32 \mathrm{~K}$ data points of $2.1 \mathrm{sec}$ and a spectral width of 7,500 Hz [31]. The FID signal was measured with an exponential line broadening of $0.3 \mathrm{~Hz}$ and was Fourier-transformed to obtain the ${ }^{1} \mathrm{H}-$ NMR spectra. The broad NMR signals of macromolecules like lipoproteins and proteins were reduced with a fixed total spin-echo delay $(2 \mathrm{n} \tau)$ of $40 \mathrm{msec} .{ }^{1} \mathrm{H}-\mathrm{NMR}$ spectra were manually phased and baseline-corrected using TopSpin software version 3.0 (Bruker Biospin, Rheinstetten, Germany).

Pre-processing of NMR spectral data: Raw NMR data were processed as previously described $[9,30]$. The ${ }^{1} \mathrm{H}-$ NMR spectra were corrected by the TSP signal into $\delta_{\mathrm{H}} 0.00$ and automatically converted into ASCII files using MestReNova Version 8.0.1 (Mestrelab Research SL, Santiago de Compostela, Spain). The ASCII files were imported into "R" software (http://cran.r-project.org/) and aligned using an open-access software package. The spectra were binned into consecutive non-overlapping regions of $\delta 0.003$ chemical 
Table 2. Assignment of the identified metabolites in serum of healthy cows and cows with footrot

\begin{tabular}{rllc}
\hline No. Metabolites & \multicolumn{1}{c}{ Chemical shift $\delta{ }^{1} \mathrm{H}^{\mathrm{a})}$} & $\begin{array}{c}\text { Expression } \\
\left.\text { change }{ }^{\mathrm{b}}\right)\end{array}$ \\
\hline 1 & 3-Hydroxy-3-methylglutarate & $1.32(\mathrm{~s}), 2.39(\mathrm{~d}), 2.45(\mathrm{~d})$ & $\uparrow$ \\
2 & 3-Hydroxybutyrate & $1.20(\mathrm{~d}), 2.30(\mathrm{dd}), 2.40(\mathrm{dd}), 4.13(\mathrm{dd})$ & $\uparrow$ \\
3 & 3-Hydroxyisobutyrate & $1.05(\mathrm{~d}), 2.46(\mathrm{~m}), 3.53(\mathrm{q}), 3.69(\mathrm{q})$ & $\downarrow$ \\
4 & Ethanol & $1.19(\mathrm{t}), 3.67(\mathrm{q})$ & $\downarrow$ \\
5 & Acetoacetate & $2.28(\mathrm{~s}), 3.44(\mathrm{~s})$ & $\downarrow$ \\
6 & Alanine & $1.49(\mathrm{~d}), 3.78(\mathrm{dd})$ & $\downarrow$ \\
7 & Acetone & $2.24(\mathrm{~s})$ & $\downarrow$ \\
8 & Carnitine & $2.40(\mathrm{q}), 2.44(\mathrm{dd}), 3.21(\mathrm{~s}), 3.38(\mathrm{t}), 3.43(\mathrm{t})$ & $\downarrow$ \\
9 & Pyruvate & $2.38(\mathrm{~s})$ & $\downarrow$ \\
10 & Sarcosine & $2.74(\mathrm{~s}), 3.61(\mathrm{~s})$ & $\downarrow^{*}$ \\
11 & Succinate & $2.39(\mathrm{~s})$ & $\downarrow$ \\
12 & Betaine & $3.25(\mathrm{~s}), 3.89(\mathrm{~s})$ & $\downarrow$ \\
13 & Creatine & $3.04(\mathrm{~s}), 3.93(\mathrm{~s})$ & $\downarrow$ \\
14 & 1-Methylhistidine & $3.66(\mathrm{~s})$ & $\downarrow$ \\
15 & Glycine & $3.55(\mathrm{~s})$ & $\downarrow$ \\
16 & Glucose & $3.26(\mathrm{dd}), 3.41(\mathrm{t}), 3.45(\mathrm{~m}), 3.8(\mathrm{t})$ & $\downarrow$ \\
17 & Glycerate & $3.7(\mathrm{dd})$ & $\uparrow^{* *}$ \\
18 & Lactate & $1.33(\mathrm{~d}), 4.12(\mathrm{q})$ & $\downarrow$ \\
19 & Serine & $3.84(\mathrm{dd}), 3.96(\mathrm{~m})$ & $\downarrow^{* *}$ \\
20 & Valine & $0.99(\mathrm{~d}), 1.02(\mathrm{~d}), 3.62(\mathrm{~d})$ & $\downarrow$ \\
21 & 3 -Methylhistidine & $3.71(\mathrm{~s}), 3.92(\mathrm{dd})$ & \\
\hline
\end{tabular}

a) Multiplicity: s, singlet peak; d, doublet peaks; dd, double doublet peaks; t, triplet peaks; q, quartet peak; m, multiple peaks. b) " $\uparrow$ " and " $\downarrow$ " represent increased and decreased levels in the footrot cows compared with control cows, respectively. “*” and “**” represent significant differences $(P<0.05)$ and highly significant differences $(P<0.01)$, respectively.

shift bins between $\delta$ 0.4-9.0 to avoid the encompassment of multiple peaks. The chemical shift at $\delta 0.0$ was referenced to the TSP signal. Regions of residual water and EDTA signals between $\delta$ 4.2-9.0 were removed. Based on least square minimization, the remaining binned data $(\delta 0.4-4.2)$ of each NMR spectrum were auto-scaled and mean-centered to reduce multicollinearity and to simplify the interpretation of the modeling coefficients. For each spectrum, we calculated the integral values for different sample dilutions to reduce the effects of any concentration variability among the samples.

Multivariate pattern recognition: Multivariate statistical analyses of NMR data were performed by SIMCA-P 15.0 software (Umetrics, UmeÅ, Sweden). Unsupervised principal component analysis (PCA) and supervised orthogonal partial least-squares discriminant analysis (OPLS-DA) were used to determine differences between groups.

Based on a Hotelling's T2 range plot, PCA was performed on the mean-centered data to identify possible outliers and to assess any intrinsic clustering from the two groups [36]. A PCA model was performed with the first two principal components (PC1 and PC2) in the scores plot. Through an orthogonal filter, the OPLS-DA model was used to identify the most significant variations between the two groups and remove unrelated systematic variations. A 10-fold crossvalidated method was performed in the OPLS-DA model. In the scores plot of OPLS-DA, t[1]P represents the first principal component, and $\mathrm{t}[2] \mathrm{O}$ represents the second orthogonal component. $\mathrm{R}^{2}$ and $\mathrm{Q}^{2}$, which represent the predicted ability of the model and the explained variability, respectively, were used to describe the quality of the OPLS-DA model. A colorcoded coefficient plot of the OPLS-DA model was generated to identify differential metabolites between the two groups. In color-coded coefficient plots, red signals in the spectra corresponded to metabolites with greater contribution to separation between the two groups than blue signals. Signals with a positive or negative direction of $\mathrm{t}[1] \mathrm{P}$ corresponded to metabolites that were present at high or low concentrations, respectively.

Identification of metabolites and metabolic pathway analysis: Based on the chemical shifts, metabolites in the two groups were identified with the Chenomx NMR Suite 7.5 library (Chenomx Inc., Edmonton, Canada). The identified metabolites were defined based on data from a previous study and public databases, e.g., the Human Metabolome Database (HMDB, http://www.hmdb.ca) and the Biological Magnetic Resonance Data Bank (BMRB, www.bmrb. wisc.edu) [24]. Serum metabolites from the two groups are presented in Table 2. Based on the Kyoto Encyclopedia of Genes and Genomes (KEGG, http://www.kegg.jp), the impact factor of the topology analyses of metabolic pathways was graphically presented using metabolomics data analysis software (Metaboanalyst 2.0, www.metaboanalyst. ca/MetaboAnalysts) [32]. The results were presented graphically as a bubble plot. Darker colored, larger areas of the bubbles represent more significant metabolite changes in the corresponding pathway. 
Univariate analysis: Clinical data, serum biochemistry data and differential metabolites between the two groups were analyzed by one-way ANOVA using SPSS statistical software Version 11.0 (SPSS, Inc., Chicago, IL, U.S.A.). Data were presented as mean \pm standard error of the mean (SEM). $P<0.05$ was considered statistically significant.

\section{RESULTS}

Clinical information and serum biochemistry analysis: The clinical data and serum biochemical differences between cows with footrot (F group) and the healthy controls (C group) are shown in Table 1. There were no differences in age, parity or DMI between the two groups $(P>0.05)$. On the other hand, BCS was significantly different between $\mathrm{F}$ and $\mathrm{C}$ groups $(P<0.05)$. Serum Glc, NO, T-AOC, SOD and GSH-Px were significantly different between the two groups $(P<0.05)$. No significant differences were observed for TG, ALT, AST, TP or MDA levels $(P>0.05)$.

Metabolic profiles of serum samples from dairy cows: The ${ }^{1} \mathrm{H}-\mathrm{NMR}(\delta 0.4-4.2)$ spectra revealed a wide range of metabolites. Typical CPMG ${ }^{1} \mathrm{H}-\mathrm{NMR}$ profiles of sera obtained from the $\mathrm{F}$ and $\mathrm{C}$ groups are presented in Fig. 1. Twenty-one metabolites were identified and confirmed by public databases and two-dimensional NMR techniques (heteronuclear single quantum correlation and total correlation spectroscopy). The endogenous metabolites included glucose, ketone bodies (acetone, 3-hydroxybutyrate, 3-hydroxy3-methylglutarate, 3-hydroxyisobutyrate and acetoacetate) and amino acids (alanine, serine, valine, glycine, sarcosine, 1-methylhistidine and 3-methylhistidine), among others. The main differential metabolites were identified through multivariate data analysis.

Determining metabolic variations between groups using multivariate analysis: First, a PCA scores plot with pareto-scaled data was generated and analyzed (Fig. 2). PC1 and PC2 accounted for $43.91 \%$ and $14.49 \%$ of the total variation in the dataset, respectively. The OPLS-DA model was performed to discriminate samples according to their groups (footrot or control). The results are shown in Fig. 3. The OPLS-DA score plot (Fig. 3a) demonstrated a reasonable and considerable separation between the two groups $\left(\mathrm{R}^{2}=0.79 ; \mathrm{Q}^{2}=0.75\right)$, suggesting a significant metabolic perturbation in the footrot group. To identify the 21 serum metabolites that contributed to group separation, OPLS-DA color-coded loadings plots (Fig. 3b and c) were generated. For each variable, information was provided by different colors (red representing high values; blue representing low values), based on the absolute correlation coefficient $(|\mathrm{r}|)$. The significant metabolites in the negative regions of the loading plot (Fig. 3b and 3c) were increased in cows with footrot. Conversely, the significant metabolites in the positive regions of the loading plot were decreased in cows with footrot. The results of OPLS-DA revealed that 21 different metabolites were present (Table 2). The OPLS-DA loading plot showed that cows with footrot had higher levels of 3-hydroxy-3-methylglutarate, 3-hydroxybutyrate and lactate, and lower levels of 3-hydroxyisobutyrate, ethanol, acetoacetate, alanine, acetone, carnitine, betaine, creatine, isoleucine, glycine, glucose, glycerate, serine, 3-methylhistidine, pyruvate, sarcosine, succinate and valine, compared to the control cows. The $P$-values of the 21 metabolites were obtained by $t$-tests (Table 2). Levels of pyruvate and sarcosine $(P<0.05)$, and lactate, succinate and valine $(P<0.01)$ were significantly different between cows from $\mathrm{F}$ and $\mathrm{C}$ groups.

Identification of impacted metabolite pathways: The MetaboAnalyst 2.0 software was used to provide impact factors from the pathway topology analysis for all analyzed pathways. The 28 different metabolic pathways of the obtained serum metabolites were mapped (Fig. 4). The top 10 pathways for footrot included glycine, serine and threonine metabolism, ketone body synthesis and degradation, methane metabolism, valine, leucine and isoleucine biosynthesis, pyruvate metabolism, aminoacyl-tRNA biosynthesis, glycerolipid metabolism, butanoate metabolism, glycolysis or gluconeogenesis, and the TCA cycle (Table 3).

\section{DISCUSSION}

Dairy cows with acute footrot can cause considerable economic loss through a reduction in milk production [21]. If not treated in time, the infection can lead to blood poisoning and be life threatening [19]. Footrot is a multifactorial disease related to immune, hormonal, nutritional and environmental factors as well as infection [16]. However, a comprehensive study of the etiological effects of these causative factors in the pathogenesis of footrot has not yet been performed. Metabolomics provides a novel pathway for resolving this question. ${ }^{1} \mathrm{H}-\mathrm{NMR}$-based metabolomics represents a powerful tool for identifying serum metabolites in ruminant animals $[3,22]$. Further, preliminary studies by our group suggested that ${ }^{1} \mathrm{H}-\mathrm{NMR}$-based metabolomics can assess ketosis and milk fever in dairy cows and identify metabolites [28, 29]. However, little is known about the metabolic impact of footrot in dairy cows. In this study, an ${ }^{1} \mathrm{H}-\mathrm{NMR}$-based metabolomics approach combined with biochemical assays was used to comprehensively assess serum metabolites in dairy cows with and without footrot.

Antioxidants play an important physiological role in counteracting free radicals and preventing cellular damage. The TAC was measured to determine the cumulative action of all the antioxidants present in blood. A significant decrease $(P<0.001)$ in TAC level was detected in cows with footrot compared to controls. The GSH-Px activity was significantly lower $(P<0.001)$ in the $\mathrm{F}$ group compared to the controls, which suggests a reduction in antioxidative protection and superoxide radical scavenging action in cows with footrot. The high level of serum SOD seen in the F group may be a compensatory response to protect the body from oxidative damage. These observations may be because of alterations in the antioxidative and oxidative balance, which is in accordance with a previous study [1]. Activation of the immune system by footrot infection will release reactive oxygen and reactive nitrogen species, inducing oxidative stress and lipid peroxidation, which affects cow development [14]. MDA is produced by lipid peroxide, which is used as a marker for 


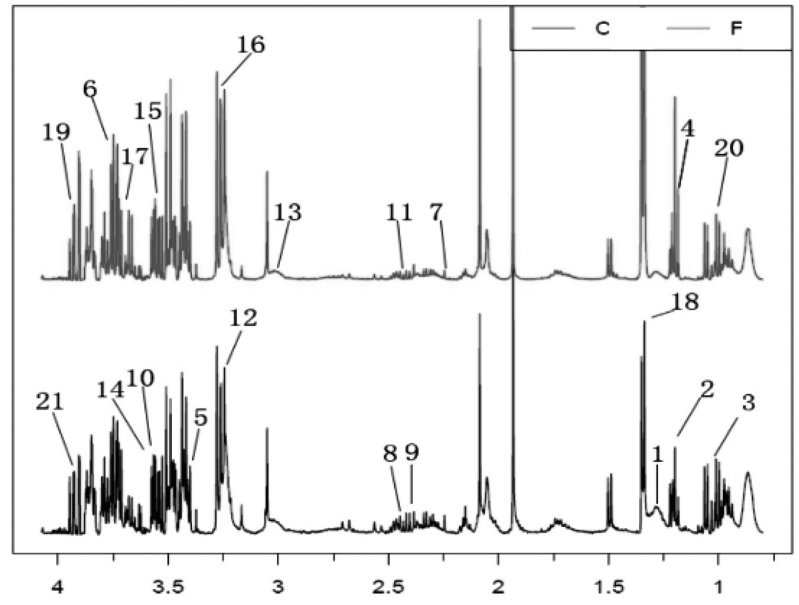

Fig. 1. Typical $500 \mathrm{MHz}{ }^{1} \mathrm{H}-\mathrm{NMR}$ spectrum of serum samples from footrot (F, top spectrum) and control (C, bottom spectrum) and footrot (F, spectrum) groups. A total of 21 metabolites were assigned. Metabolites: 1, 3-hydroxy-3-methylglutarate; 2, 3-hydroxybutyrate; 3, 3-hydroxyisobutyrate; 4, ethanol; 5, acetoacetate; 6 , alanine; 7 , acetone; 8 , carnitine; 9 , pyruvate; 10 , sarcosine; 11 , succinate; 12 , betaine; 13 , creatine; 14,1 -methylhistidine; 15 , glycine; 16 , glucose; 17 , glycerate; 18 , lactate; 19 , serine; 20 , valine; 21, 3-methylhistidine.

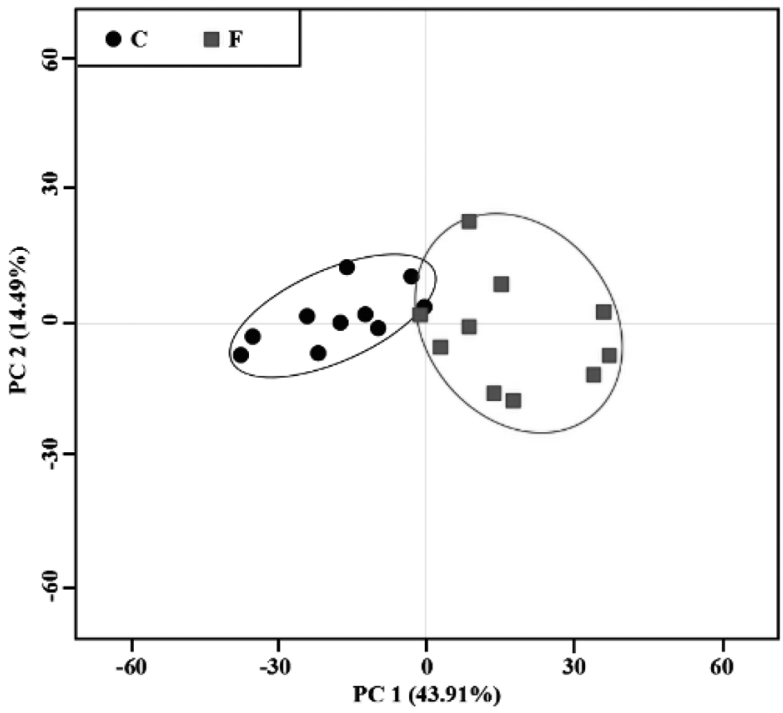

Fig. 2. PCA score plot of ${ }^{1} \mathrm{H}-\mathrm{NMR}$ spectra of serum samples from cows with footrot (box, - ) and healthy controls (dot, •). Each point represents an individual sample on the score plot. The center and margin of the ellipse represent mean and standard deviation, respectively.
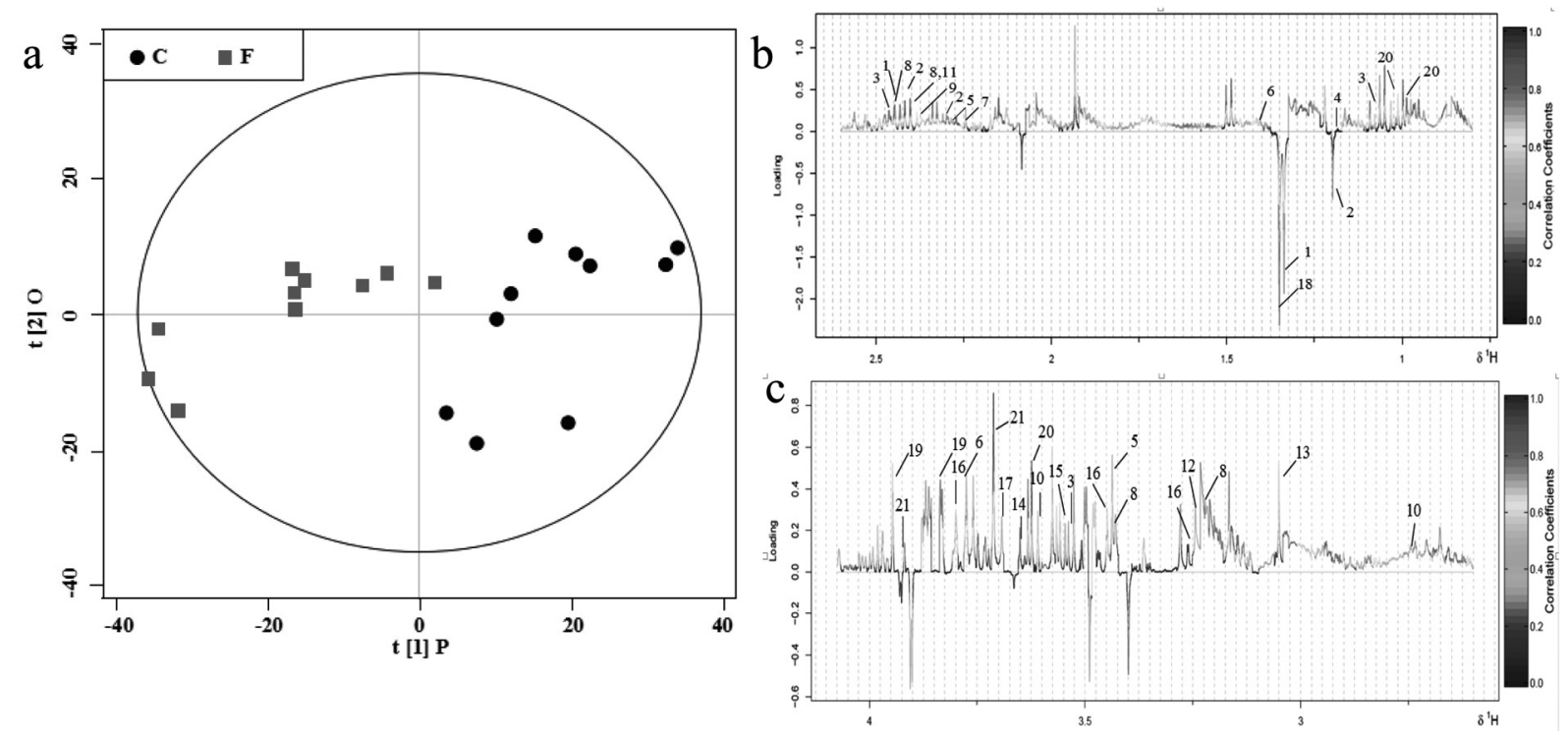

Fig. 3. OPLS-DA comparison of serum spectra between the $\mathrm{C}$ and $\mathrm{F}$ groups. (a) OPLS-DA scores plot obtained from ${ }^{1} \mathrm{H}-\mathrm{NMR}$ CPMG spectral data for cows with footrot $(\mathrm{F}$, box, $\mathbf{m})$ and controls $(\mathrm{C}$, dot, $\bullet$ ), where one point represents one sample. (b and c) Corresponding loading plots $(\delta 0.4-2.5$ and 2.6-4.2) color-coded according to correlation coefficient from blue to red. The upward and downward peaks represent decreased and increased levels in cows with footrot, respectively. The metabolites are presented in Table 2.

oxidative damage induction [20]. Higher levels of serum MDA indicated lipid peroxidation in cows with footrot. Large quantities of NO, synthesized by infectious diseaseactivated macrophages, play a major role in defense mechanisms [15]. NO also may cause tissue damage during inflammation, through the formation of peroxynitrite. Peroxynitrite is a powerful oxidant capable of initiating lipid peroxidation [4]. Significantly elevated NO levels in cows with footrot $(P<0.001)$ suggest that lipid peroxidation is induced in this disease. Furthermore, liver damage is often induced under conditions of lipid peroxidation, as shown by a slight rise in serum levels of AST and ALT [32]. Higher AST levels 
Table 3. Significantly altered metabolic pathways in cows with footrot

\begin{tabular}{clcccc}
\hline No. & \multicolumn{1}{c}{ Pathway } & Metabolites $^{\mathrm{a})}$ & Hits $^{\mathrm{b})}$ & $-\log (\mathrm{g})$ & Impact \\
\hline 1 & Glycine, serine and threonine metabolism & 32 & 7 & 15.66 & 0.596 \\
2 & Synthesis and degradation of ketone bodies & 5 & 2 & 6.16 & 0.600 \\
3 & Methane metabolism & 9 & 2 & 4.92 & 0.400 \\
4 & Valine, leucine and isoleucine biosynthesis & 11 & 2 & 4.51 & 0.338 \\
5 & Pyruvate metabolism & 22 & 2 & 3.17 & 0.189 \\
6 & Aminoacyl-tRNA biosynthesis & 64 & 3 & 2.68 & 0.140 \\
7 & Glycerolipid metabolism & 18 & 1 & 1.42 & 0.105 \\
8 & Butanoate metabolism & 20 & 4 & 8.74 & 0.101 \\
9 & Glycolysis or Gluconeogenesis & 26 & 3 & 5.09 & 0.099 \\
10 & Citrate cycle (TCA cycle) & 20 & 2 & 3.35 & 0.098 \\
\hline
\end{tabular}

a) Total number of compounds in pathways based on KEGG databases. b) Matched number of identified metabolites in serum from $\mathrm{F}$ and $\mathrm{C}$ groups.

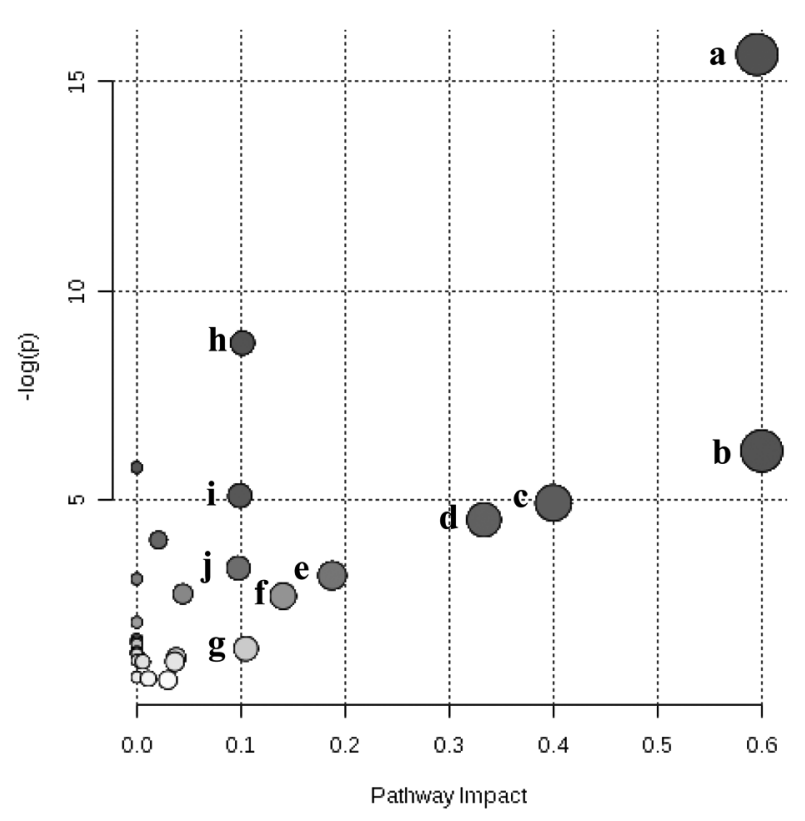

Fig. 4. The bubble plot shows a metabolomic overview of altered metabolic pathways, mapping serum metabolites identified in footrot and control groups. Keys: (a) glycine, serine and threonine metabolism; (b) synthesis and degradation of ketone bodies; (c) methane metabolism; (d) valine, leucine and isoleucine biosynthesis; (e) pyruvate metabolism; (f) aminoacyl-tRNA biosynthesis; (g) glycerolipid metabolism; (h) butanoate metabolism; (i) glycolysis or gluconeogenesis; and (j) citrate cycle (TCA cycle).

were observed in cows with footrot, which may be attributed to an acute dystrophic muscle condition as described by a previous study [38].

Combined with statistical models, ${ }^{1} \mathrm{H}-\mathrm{NMR}$ identified alterations in specific metabolic pathways of cows with footrot. Footrot affected several metabolic pathways, including amino acid metabolism, carbohydrate metabolism, lipid metabolism and energy metabolism.

Alterations in amino acid metabolism: Cows with footrot had significant disturbances in amino acid metabolism. This was evident from the low levels of valine, betaine, glycine, alanine, creatine, serine, sarcosine, carnitine, ethanol, 1-methylhistidine and 3-hydroxyisobutyrate. Past studies by our group showed that the blood levels of 1-methylhistidine, 3-hydroxyisobutyrate, alanine, betaine, creatine and ethanol were also lower in dairy cows with ketosis, milk fever or fatty liver than in controls [28-29, 35]. Meanwhile, the blood levels of glycine and valine in cows with footrot and other diseases (ketosis and fatty liver) showed the opposite trend. Valine, glycine, 3-hydroxyisobutyrate (an intermediate of valine metabolism) and serine are gluconeogenic amino acids that produce oxaloacetate [12]. Alanine, a non-essential amino acid that can be synthesized from pyruvate, is one of the most important energy sources released by muscle tissues. Footrot often affects animal physiology in many ways by causing mild fever, lameness, severe pain and a resulting drop in feed intake. However, this study was performed on acute cases of footrot in cows. Compared with the control cows, the DMI showed an insignificant downward trend $(P=0.058)$ in cows with footrot. Based on the results, the lack of gluconeogenic substrates may play an important role in the pathogenesis of acute footrot. Creatine increases the synthesis of adenosine triphosphate (ATP) in all body cells. Additionally, creatine plays a vital role in the urea cycle and in ATP homeostasis [33]. Decreased creatine levels suggest lack in energy of cows with footrot.

Sarcosine, an intermediate in the conversion of choline to glycine, is a protein constituent [2]. Additionally, sarcosine is a prime metabolic source of components like creatine and serine for living cells. Serum biochemistry analysis showed that the level of TP in F group serum was slightly decreased compared to the level in $\mathrm{C}$ group serum. Low levels of serum sarcosine in cows with footrot indicated high protein utilization.

1-Methylhistidine, which is converted into histidine, is closely linked to inflammation, oxidative stress and proteinenergy wasting. 3-Methylhistidine, a product of peptide bond synthesis actin and myosin methylation, is an indicator of muscle protein catabolism. Previous studies have shown that 3-methylhistidine is a clinical marker of nitrogen loss, occurring in conditions, such as trauma and infection [26]. Changes of 1-methylhistidine and 3-methylhistidine levels in cows with footrot suggest insufficient dietary protein in- 
take and high oxidative stress.

Alterations in carbohydrate metabolism: Compared with healthy controls, cows with footrot had reduced glucose and pyruvate levels. Glucose plays an important role in oxidation/reduction, and pyruvate is a key compound in the network of metabolic processes [5]. Glucose produces ATP via glycolysis, a pathway in which glucose is converted into pyruvate. Under aerobic conditions, pyruvate is subsequently converted into acetyl $\mathrm{CoA}$, the compound that initiates the TCA cycle. Cows with footrot have reduced ATP synthesis from glycolysis, glycogenolysis and the TCA cycle. On the other hand, lactate is produced as a waste product during the process of converting glucose into pyruvate. Lactate is metabolized to form glucose, by the process known as gluconeogenesis. However, because of enzymes' high affinity for glucose, a high rate of glycolysis was maintained even in the presence of oxygen, leading to increased lactate levels $[7,38]$. In the present study, higher serum lactate levels in cows with footrot may be related to the inhibition of glycolysis and gluconeogenesis, indicating inefficient metabolism [37]. In contrast, decreased lactate levels were found in our study on cows with ketosis and fatty liver, which indicated an increase in gluconeogenesis [28, 35].

Phosphate derivatives of glycerate, such as 1,3-bisphosphoglyceric acid, 2-phosphoglyceric acid, 2,3-bisphosphoglyceric acid and 3-phosphoglyceric acid, are important biochemical intermediates of glycolysis. In humans, lack of glycerate kinase leads to D-glyceric aciduria and D-glycerate anemia, which contribute to failure to thrive, progressive neurological impairment, seizures, hypotonia and metabolic acidosis [25]. In this study, cows with footrot had low levels of serum glycerate, which revealed the presence of a carbohydrate metabolic disorder.

Succinate is an important biochemical intermediate, playing an important role in the TCA cycle. Furthermore, succinate is capable of donating electrons to the electron transport chain [32]. Low levels of serum succinate in cows with footrot demonstrated a reduced flux through the TCA cycle. However, we found that blood succinate levels were increased in a fatty-liver study by our group. This result suggests that the TCA cycle is upregulated in body glucose metabolism [28].

Alterations in lipid metabolism: Several studies have shown that carnitine plays an important role in fatty acid metabolism [11]. Carnitine increases fatty acid transport into mitochondria, thereby increasing pyruvate oxidation. Decreased carnitine levels were observed in cows with footrot, indicating enhanced fatty acid transport into mitochondria.

Ketone bodies (i.e., acetoacetate, 3-hydroxybutyrate and acetone) derived from acetyl-CoA are byproducts of fatty acid oxidation [28]. Cows with footrot had reduced levels of acetoacetate and acetone, and high levels of 3-hydroxybutyrate, indicative of increased lipolysis. As a hydroxy fatty acid, 3-hydroxy-3-methylglutarate inhibits cholesterol synthesis in non-insulin-dependent diabetics [18]. In this experiment, cows with footrot had elevated levels of 3-hydroxy-3-methylglutarate.

${ }^{1} \mathrm{H}-\mathrm{NMR}$ metabolomic profiling was performed on serum to explore metabolic alterations in cows with footrot. Footrot affected amino acid, carbohydrate and lipid metabolic processes. The serum levels of four metabolites were found to be significantly different $(P<0.05)$ between cows with footrot and healthy controls. These metabolites may be useful for diagnosing acute or continuous impaired nutritional conditions in cows with footrot. Metabolomics provides a novel viewpoint and technological approach to link disease processes with biochemical pathways. ${ }^{1} \mathrm{H}-\mathrm{NMR}$ techniques can reveal the functions of different body systems. In summary, serum metabolite profiling demonstrated significant differences between cows with footrot and healthy controls.

ACKNOWLEDGMENTS. The authors report no conflicts of interest. The authors alone are responsible for the content and preparation of the manuscript. The project was supported by grants from the Natural Science Foundation of Heilongjiang Province of China (Grant Number: C201229) and the National Key Technology R\&D Program of China (Grant Numbers: 2012BAD12B05-2 and 2013BAD21B01). We are grateful to the members of Hongbin Wang's laboratory who contributed to this research.

\section{REFERENCES}

1. Al-Qudah, K. M. and Ismail, Z. B. 2012. The relationship between serum biotin and oxidant/antioxidant activities in bovine lameness. Res. Vet. Sci. 92: 138-141. [Medline] [CrossRef]

2. Allen, R. H., Stabler, S. P. and Lindenbaum, J. 1993. Serum betaine, N,N-dimethylglycine and $\mathrm{N}$-methylglycine levels in patients with cobalamin and folate deficiency and related inborn errors of metabolism. Metabolism 42: 1448-1460. [Medline] [CrossRef]

3. Başoğlu, A., Başpinar, N. and Coşkun, A. 2014. Nmr-based metabolomic evaluation in dairy cows with displaced abomasum. Turk. J. Vet. Anim. Sci. 38: 325-330. [CrossRef]

4. Beckman, J. S., Beckman, T. W., Chen, J., Marshall, P. A. and Freeman, B. A. 1990. Apparent hydroxyl radical production by peroxynitrite: implications for endothelial injury from nitric oxide and superoxide. Proc. Natl. Acad. Sci. U.S.A. 87: 1620-1624. [Medline] [CrossRef]

5. Bruck, R., Aeed, H., Schey, R., Matas, Z., Reifen, R., Zaiger, G., Hochman, A. and Avni, Y. 2002. Pyrrolidine dithiocarbamate protects against thioacetamide-induced fulminant hepatic failure in rats. J. Hepatol. 36: 370-377. [Medline] [CrossRef]

6. Bruijnis, M. R., Beerda, B., Hogeveen, H. and Stassen, E. N. 2012. Assessing the welfare impact of foot disorders in dairy cattle by a modeling approach. Animal 6: 962-970. [Medline] [CrossRef]

7. Carpenter, K. L. H., Jalloh, I. and Hutchinson, P. J. 2015. Glycolysis and the significance of lactate in traumatic brain injury. Front. Neurosci. 9: 112. [Medline] [CrossRef]

8. Dhungyel, O., Hunter, J. and Whittington, R. 2014. Footrot vaccines and vaccination. Vaccine 32: 3139-3146. [Medline] [CrossRef]

9. Dong, G., Wei, D., Wang, J., Guo, P., Li, M., Yang, M. and Kong, L. 2015. Study of the cardiotoxicity of Venenum Bufonis in rats using an 1H NMR-based metabolomics approach. PLOS ONE 10: e0119515. [Medline] [CrossRef]

10. Draper, H. H. and Hadley, M. 1990. Malondialdehyde determination as index of lipid peroxidation. Methods Enzymol. 186: 
421-431. [Medline] [CrossRef]

11. Ferrari, R., Merli, E., Cicchitelli, G., Mele, D., Fucili, A. and Ceconi, C. 2004. Therapeutic effects of L-carnitine and propionyl-L-carnitine on cardiovascular diseases: a review. Ann. N. Y. Acad. Sci. 1033: 79-91. [Medline] [CrossRef]

12. Gerich, J. E., Meyer, C., Woerle, H. J. and Stumvoll, M. 2001. Renal gluconeogenesis: its importance in human glucose homeostasis. Diabetes Care 24: 382-391. [Medline] [CrossRef]

13. Guzel, M., Askar, T. K., Kaya, G., Atakisi, E. and Avci, G. E. 2008. Serum sialic acids, total antioxidant capacity, and adenosine deaminase activity in cattle with theileriosis and anaplasmosis. Bull. Vet. Inst. Pulawy 52: 227-230.

14. Halliwell, B., Gutteridge, J. M. and Cross, C. E. 1992. Free radicals, antioxidants, and human disease: where are we now? $J$. Lab. Clin. Med. 119: 598-620. [Medline]

15. Kaur, H. and Halliwell, B. 1994. Evidence for nitric oxide-mediated oxidative damage in chronic inflammation. Nitrotyrosine in serum and synovial fluid from rheumatoid patients. FEBS Lett. 350: 9-12. [Medline] [CrossRef]

16. Kennan, R. M., Han, X., Porter, C. J. and Rood, J. I. 2011. The pathogenesis of ovine footrot. Vet. Microbiol. 153: 59-66. [Medline] [CrossRef]

17. Kumari, K., Kumar, K. and Pravin, P. K. 2013. Clinical management of foot rot in bovines. Intas Polivet 14: 78-79.

18. Frank, J., Eliasson, C., Leroy-Nivard, D., Budek, A., Lundh, T., Vessby, B., Aman, P. and Kamal-Eldin, A. 2004. Dietary secoisolariciresinol diglucoside and its oligomers with 3-hydroxy3-methyl glutaric acid decrease vitamin E levels in rats. Br. $J$. Nutr. 92: 169-176. [Medline] [CrossRef]

19. Greenough, P. R. 2007. Bovine Laminitis and Lameness. WB Saunders, Philadelphia. pp. 199-205.

20. Kandemir, F. M., Issi, M., Benzer, F., Gul, Y., Basbug, O. and Ozdemir, N. 2011. Plasma nitric oxide concentrations and erythrocyte arginase activities in lambs with contagious ecthyma. Revue Med. Vet. 162: 275-278.

21. Kausche, F. M. and Robb, E. J. 2003. A comprehensive review of ceftiofur sodium and hydrochloride formulations for treatment of acute bovine foot rot. Vet. Ther. 4: 83-93. [Medline]

22. Klein, M. S., Almstetter, M. F., Schlamberger, G., Nürnberger, N., Dettmer, K., Oefner, P. J., Meyer, H. H., Wiedemann, S. and Gronwald, W. 2010. Nuclear magnetic resonance and mass spectrometry-based milk metabolomics in dairy cows during early and late lactation. J. Dairy Sci. 93: 1539-1550. [Medline] [CrossRef]

23. Nagaraja, T. G., Narayanan, S. K., Stewart, G. C. and Chengappa, M. M. 2005. Fusobacterium necrophorum infections in animals: pathogenesis and pathogenic mechanisms. Anaerobe 11: 239-246. [Medline] [CrossRef]

24. Nicholson, J. K., Lindon, J. C. and Holmes, E. 1999. 'Metabonomics': understanding the metabolic responses of living systems to pathophysiological stimuli via multivariate statistical analysis of biological NMR spectroscopic data. Xenobiotica 29: 1181-1189. [Medline] [CrossRef]

25. Sass, J. O., Fischer, K., Wang, R., Christensen, E., Scholl-Bürgi, S., Chang, R., Kapelari, K. and Walter, M. 2010. D-glyceric aciduria is caused by genetic deficiency of D-glycerate kinase
(GLYCTK). Hum. Mutat. 31: 1280-1285. [Medline] [CrossRef] 26. Sjölin, J., Stjernström, H., Henneberg, S., Hambraeus, L. and Friman, G. 1989. Evaluation of urinary 3-methylhistidine excretion in infection by measurements of 1-methylhistidine and the creatinine ratios. Am. J. Clin. Nutr. 49: 62-70. [Medline]

27. Sun, D., Zhang, H., Guo, D., Sun, A. and Wang, H. 2013. Shotgun proteomic analysis of plasma from dairy cattle suffering from footrot: characterization of potential disease-associated factors. PLOS ONE 8: e55973. [Medline] [CrossRef]

28. Sun, L. W., Zhang, H. Y., Wu, L., Shu, S., Xia, C., Xu, C. and Zheng, J. S. 2014. (1)H-Nuclear magnetic resonance-based plasma metabolic profiling of dairy cows with clinical and subclinical ketosis. J. Dairy Sci. 97: 1552-1562. [Medline] [CrossRef]

29. Sun, Y., Xu, C., Li, C., Xia, C., Xu, C., Wu, L. and Zhang, H. 2014. Characterization of the serum metabolic profile of dairy cows with milk fever using $1 \mathrm{H}-\mathrm{NMR}$ spectroscopy. Vet. Q. 34: 159-163. [Medline] [CrossRef]

30. Tian, N., Wang, J. S., Wang, P. R., Song, X. F., Yang, M. H. and Kong, L. Y. 2013. Nmr-based metabonomic study of chinese medicine gegen qinlian decoction as an effective treatment for type 2 diabetes in rats. Metabolomics 9: 1228-1242. [CrossRef]

31. Viant, M. R., Rosenblum, E. S. and Tieerdema, R. S. 2003. NMR-based metabolomics: a powerful approach for characterizing the effects of environmental stressors on organism health. Environ. Sci. Technol. 37: 4982-4989. [Medline] [CrossRef]

32. Wei, D. D., Wang, J. S., Wang, P. R., Li, M. H., Yang, M. H. and Kong, L. Y. 2014. Toxic effects of chronic low-dose exposure of thioacetamide on rats based on NMR metabolic profiling. $J$. Pharm. Biomed. Anal. 98: 334-338. [Medline] [CrossRef]

33. Wei, D. D., Ge-Dong., Guo, P. P., Wang, J. S., Li, M. H., Yang, M. H. and Kong, L. Y. 2014. A bird's eye view of anisatin induced convulsive seizures in brain by a (1)H NMR based metabolic approach. Mol. Biosyst. 10: 2923-2934. [Medline]

34. Wildman, E. E., Jones, G. M., Wagner, P. E., Boman, R. L., Troutt, H. F. and Lesch, T. N. 1982. A dairy cow body condition scoring system and its relationship to selected production characteristics. J. Dairy Sci. 65: 495-501. [CrossRef]

35. Xu, C., Sun, L. W., Xia, C., Zhang, H. Y., Zheng, J. S. and Wang, J. S. 2016. 1h-nuclear magnetic resonance-based plasma metabolic profiling of dairy cows with fatty liver. Asian-australas. $J$. Anim. Sci. 29: 219-229. [Medline] [CrossRef]

36. Yde, C. C., Clausen, M. R., Ditlev, D. B., Lillefosse, H., Madsen, L., Kristiansen, K., Liaset, B. and Bertram, H. C. 2014. Multi-block pca and multi-compartmental study of the metabolic responses to intake of hydrolysed versus intact casein in c57bl/6j mice by nmr-based metabolomics. Metabolomics 10: 938-949. [CrossRef]

37. Zhou, H., Bennett, G. and Hickford, J. G. H. 2009. Variation in Fusobacterium necrophorum strains present on the hooves of footrot infected sheep, goats and cattle. Vet. Microbiol. 135: 363-367. [Medline] [CrossRef]

38. Zhou, X. M., He, C. C., Liu, Y. M., Zhao, Y., Zhao, D., Du, Y., Zheng, W. Y. and Li, J. X. 2012. Metabonomic classification and detection of small molecule biomarkers of malignant pleural effusions. Anal. Bioanal. Chem. 404: 3123-3133. [Medline] [CrossRef] 